\title{
In vivo evaluation: the effects of a cream containing Acacia bark extract on skin melanin and erythema content
}

\author{
Atif Ali, Naveed Akhtar, Muhammad Shoaib Khan \\ Department of Pharmacy, Faculty of Pharmacy and Alternative Medicine, Islamia University of Bahawalpur, Pakistan \\ Head: Prof. Naveed Akhtar
}

Postep Derm Alergol 2012; XXIX, 5: 369-372

DOI: $10.5114 /$ pdia.2012.31490

\begin{abstract}
Introduction: Phenolic compounds have inhibitory effects on tyrosinase and inflammatory activities which lead to reduction in the melanin and erythema content. This is important for hyperpigmentation, dermatitis and other oxidative stress diseases.

Aim: Investigation of the effects of a cream containing phenolic-rich extract of Acacia bark on the human skin melanin and erythema content.

Material and methods: Eleven healthy male volunteers were analyzed and treated with active cream versus base for a period of three months by non-invasive bioengineering techniques.

Results: No serious adverse effects were observed after patch testing. Base showed insignificant results and active cream showed a significant decrease in skin melanin and erythema content after statistically applied ANOVA. Conclusions: Our results show that the Acacia bark extract represents a valuable source of phenolic compounds produced naturally. Treatments of human skin with active cream containing phenolic-rich Acacia bark extract significantly reduce skin melanin and erythema in vivo and could be used for topical treatment of hyperpigmentation and dermatitis.
\end{abstract}

Key words: Acacia nilotica, active cream, melanin, erythema.

\section{Introduction}

Investigation of the clinical aspects of depigmenting and anti-inflammatory agents is very important. Increased production and accumulation of melanin leads to many hyperpigmentation disorders such as melasma, postinflammatory pigmentation, solar lentigo, etc., which become prominent with aging [1-4]. Furthermore, plant extracts are also used in topical and cosmetic formulations such as fragrance, colorants, anti-irritant and antiaging etc [5]. Natural products may induce allergic and irritant contact dermatitis and phytophotodermatitis [6]. Topical treatments are a growing research area of formulators for skin diseases [7]. Acacia nilotica (Mimosaceae) indigenously known as 'Babul' or 'Kikar' is a proverbial, medium-sized tree and is broadly scattered in tropical and subtropical countries. Acacia nilotica is a medicinal plant acknowledged to be rich in phenolics, consisting of condensed tannin and phlobatannin, gallic acid, protocatechuic acid, pyrocatechol, (+) catechin, (-) epi-gallocate- chin-7-gallate and (-) epigallocatechin-5, 7-digallate. Different parts of this plant such as the leaves, roots, seeds, bark, fruits, flowers, gum and immature pods show anticancer, antimutagenic, spasmogenic, vasoconstrictor, antipyretic, anti-asthmatic, cytotoxic, anti-diabetic, antiplatelet aggregatory, anti-plasmodial, molluscicidal, anti-fungal and inhibitory activity against Hepatitis C virus (HCV) and human immunodeficiency virus (HIV)-I [8].

\section{Aim}

Our aim of this study was to investigate the effects of a cream containing phenolic-rich extract of Acacia bark on the human skin melanin and erythema content.

\section{Material and methods}

Acacia nilotica leaves were gathered in July 2010 in Dera Ghazi Khan, Pakistan, and air-dried at room temperature for a period of 4 weeks. Acacia nilotica bark was

Address for correspondence: Atif Ali PhD, Department of Pharmacy, Islamia University of Bahawalpur, 63100 Bahawalpur, Pakistan, phone: +923346081922, e-mail: ajmaline2000@gmail.com 
collected and identified by the Cholistan Institute of Desert studies and a specimen was deposited with the Herbarium, The Islamia University of Bahawalpur (Voucher No. AN-BK-01-01-10-030).

An active cream was prepared using an anionic hydrophilic colloid (14\% Paraffin oil), 2\% Abil EM 90, $3 \%$ Acacia nilotica bark aqueous ethanolic extract, $1 \%$ fragrance and rest of deionized water. The heated oily phase and aqueous phase were mixed using homogenizer (EuroStar, IKAD 230, Germany) by addition of the extract and fragrance. The base was prepared without the extract using the same method.

Eleven subjects aged between 20 and 35 years were selected. All subjects were healthy males with no known dermatological diseases or allergy to any substance in active creams. The Declaration of Helsinki was followed in this blind study. Informed consent was signed before the start of this study by all volunteers. The exclusion criteria were as follows: presence of any dermatitis and/or other skin or allergic diseases, smokers and previous treatment of forearms' skin with cosmetic active creams such as sunscreens, moisturizers or anti-ageing cosmetics. During the test period, the subjects were allowed to wash normally, but were instructed not to use any other skin care products on their arms. The volunteers were asked not to apply any topical products on cheeks $24 \mathrm{~h}$ before the beginning and throughout the test period. Additionally, solar exposure and use of occlusive clothes on the test area were forbidden.

Non-invasive bioengineering measurements were performed. The melanin and erythema measurements (EI) were performed with a reflectance spectrophotometer, Mexameter from Courage and Khazaka Electronic $\mathrm{GmbH}$ Cologne, Germany. The Mexameter was calibrated according to guidelines of manufacturers. All measurements were made in a draught-free room, with a controlled temperature $\left(18.0-20.6^{\circ} \mathrm{C}\right.$ ) and relative humidity (50-65\%).

For primary irritation potential of creams, patch tests were accomplished on both forearms of each volunteer on the first day of skin assessment. A $5 \mathrm{~cm} \times 4 \mathrm{~cm}$ area was marked on the forearms. The patch (Bandage disc) for the left forearm was drenched with $1.0 \mathrm{~g}$ of the base while the patch for the right forearm was drenched with $1.0 \mathrm{~g}$ of the active cream with a surgical dressing after application on marked areas. The patches were removed after $48 \mathrm{~h}$ and the forearms were observed for any skin irritation by an experienced dermatologist and also using Mexameter. A numeric scale was used to quantify the skin irritation (visual scoring). The average irritant score of the active cream was calculated from the average of the quotations obtained for each volunteer, ranking from "non-irritant" to "very irritant". The reactions were evaluated according to the following arbitrary scale: No erythema - 0, Light erythema (hardly visible) - 1, Clearly visible erythema -2 , Moderate erythema -3 , Serious erythema (dark red with possible formation of light eschars) -4 .
No edema - 0, Very light edema (hardly visible) - 1 , Light edema - 2, Moderate edema (about $1 \mathrm{~mm}$ raised skin) -3 , Strong edema (extended swelling even beyond the application area) -4 . The index of average irritation was classified according to the amended Draize system: Non irritating - 0.5, slightly irritating: 0.5-2.0, moderately irritating: 2.0-5.0, highly irritating: 5.0-8.0.

In vivo investigations have been carried out during the winter months (October to January). All instrumental measurements were made by the author according to the manufacturer's instructions. Two weeks before the study beginning and during the treatment period, the volunteers were permitted only to use normal cleansing products. Each volunteer was then given two creams, an active cream containing the extract of the plant and a base without the extract. The volunteers were wellinformed about the correct use of the creams. Measurements of skin melanin and erythema were made every second week up to the end of the study period of three months. Approximately $500 \mathrm{mg}$ of both the active cream and the base were to be applied to the cheeks twice daily (mornings: 7:00-9:00; evenings: 19:00-21:00) over a 12 weeks' period by the volunteers at home. The area around the eyes was omitted. Before all measurements, volunteers remained in the room for at least $15 \mathrm{~min}$ in order to allow for full skin adjustment to the room temperature.

To assess the effectiveness of the two creams, that is, the base and the active cream, tested in this study, the volunteers were asked to answer a questionnaire consisting of seven parameters after 3 months from the beginning of the study: 1. Ease of application, 2. Spreadability, 3. Sensation just after application, 4. Sensation in the long term, 5. Irritation, 6. Shine on skin and 7. Sensation of softness.

Skin erythema contents after application of the base and after application of the active cream were compared at the same time intervals (i.e., $0 \mathrm{~h}$ readings of erythema after application of the base were compared with $0 \mathrm{~h}$ readings of erythema after application of the active cream, $48 \mathrm{~h}$ readings of erythema after application of the base were compared with $48 \mathrm{~h}$ readings of erythema after application of the active cream).

The melanin and erythema values of the right and left cheek of the volunteers were calculated at $0 \mathrm{~h}$, in the $2^{\text {nd }}$ week, $4^{\text {th }}$ week, $6^{\text {th }}$ week, $8^{\text {th }}$ week, $10^{\text {th }}$ week and $12^{\text {th }}$ week. SPSS 17.0 was used for data analysis on the computer by using the two-way ANOVA for variation between different time intervals and the paired sample t-test for the variation between the two active creams. The level of significance was $5 \%$.

\section{Ethical standards}

The approval of this study was obtained from the Board of the Advanced Study and Research (BASAR), the Islamia University, Bahawalpur and the Institutional 
Ethical Committee, Faculty of Pharmacy and Alternative Medicine, The Islamia University, Bahawalpur.

\section{Results}

Initially, evaluation of irritancy testing was based on visual scoring only as shown in Table 1 . By performing patch testing on forearms of volunteers for $48 \mathrm{~h}$ for both the base and active cream it was found out that the erythema level after application of the base was slightly decreased while the erythema level after application of the active cream was pronouncedly decreased after $48 \mathrm{~h}$. But with a paired sample $t$-test it was evident that the effects of the active cream and base were insignificant regarding the skin erythema even though the active cream decreased the skin erythema more than the base.

In this study, slight variations were found in skin melanin values after the application of the base, but in the case of the active cream there was a continuous decrease in the skin melanin content throughout the study period. Skin melanin was determined at regular intervals on the $2^{\text {nd }}, 4^{\text {th }}, 6^{\text {th }}, 8^{\text {th }}, 10^{\text {th }}$ and $12^{\text {th }}$ week and percentage of changes are presented in Figure 1. With the help of the ANOVA test, it was found that changes in skin melanin values produced by the base were insignificant and by the active cream were significant over time. By applying the paired sample $t$-test, it was found that the active cream produced significant $(p \leq 0.05)$ effects as compared to the base.

In this study, it was found that there were slight variations observed in erythema values of the base till the $12^{\text {th }}$ week. However, for the active cream, it was found that there was a gradual decrease in erythema values up to the $12^{\text {th }}$ week (Figure 1). With the help of the ANOVA test, it was found that changes in erythema values produced by the active cream were significant and the base - were insignificant over time. When the paired sample $t$-test was applied it was found that the base and active

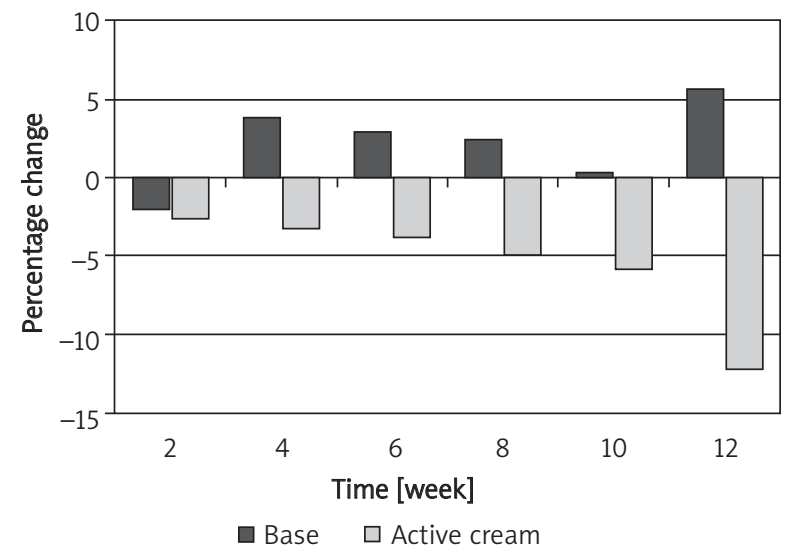

Figure 1. Percentage change in the melanin values of volunteers after the application of the base and active cream
Table 1. Values and classification of average irritation indexes

\begin{tabular}{lccc}
\hline Volunteer & Erythema & Edema & Total reading 48 h \\
\hline 1 & 0 & 0 & 0 \\
\hline 2 & 0 & 0 & 0 \\
\hline 3 & 0 & 0 & 0 \\
\hline 4 & 0 & 0 & 0 \\
\hline 5 & 0 & 0 & 0 \\
\hline 6 & 0 & 0 & 0 \\
\hline 7 & 0 & 0 & 0 \\
\hline 8 & 0 & 0 & 0 \\
\hline 10 & 0 & 0 & 0 \\
\hline 11 & 0 & 0 & 0 \\
\hline Total irritation & 0 & 0 & 0 \\
\hline Irritation index & 0.00 & 0.00 & 0.00 \\
\hline Result & 0 & 0 & Non irritant \\
\hline
\end{tabular}

cream showed significant variations regarding erythema values over time.

For efficacy assessment of creams, average points for each parameter are shown in Table 2 for both the base and active cream. In the paired sample t-test, non-significant difference between the average points for the base and active cream were observed which showed that there was no variation between the base and active cream.

\section{Discussion}

Initially, evaluation of irritancy testing was based on visual scoring. This type of evaluation, although subjective, can be a sensitive, reliable and reproducible method Patch testing after a single application is a widely used

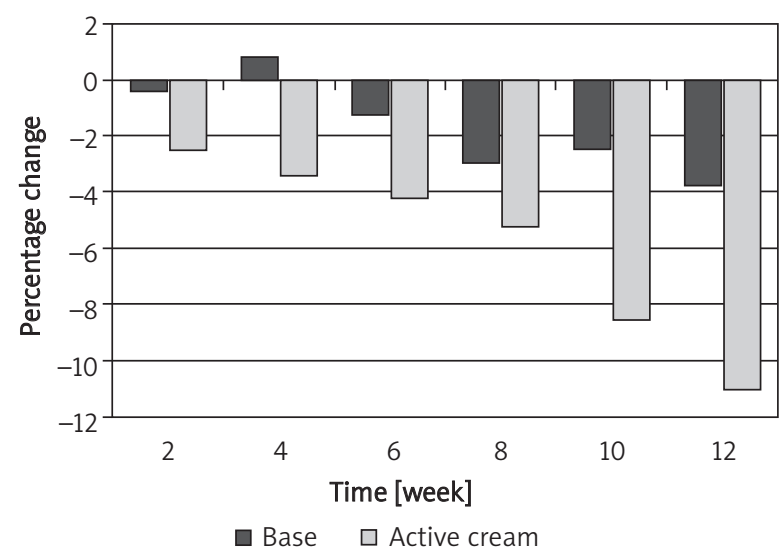

Figure 2. Percentage change in the erythema values of volunteers after the application of the base and active cream 
Table 2. Average values \pm SEM for panel test

\begin{tabular}{lcc}
\hline Variable & $\begin{array}{c}\text { Average points } \\
\text { for base } \pm \text { SEM }\end{array}$ & $\begin{array}{c}\text { Average points for } \\
\text { active cream } \pm \text { SEM }\end{array}$ \\
\hline Ease of application & $4.03 \pm 0.05$ & $4.25 \pm 0.11$ \\
\hline Spreadability & $4.16 \pm 0.08$ & $4.39 \pm 0.03$ \\
\hline $\begin{array}{l}\text { Sensation just after } \\
\text { application }\end{array}$ & $3.96 \pm 0.07$ & $3.98 \pm 0.05$ \\
\hline $\begin{array}{l}\text { Sensation in the long } \\
\text { term }\end{array}$ & $4.02 \pm 0.08$ & $4.01 \pm 0.12$ \\
\hline $\begin{array}{l}\text { Irritation } \\
\text { Shine on skin }\end{array}$ & $0.00 \pm 0.00$ & $0.00 \pm 0.000$ \\
\hline Sensation of softness & $4.41 \pm 0.08$ & $4.04 \pm 0.04$ \\
\hline
\end{tabular}

procedure to evaluate acute irritant reactions [9]. Neither erythema nor edema after the application of the base and active cream were observed. Topical applications must be safe which is shown by our results. Finally, it was concluded that both the active cream and base produced no skin irritation after performing the patch test of $48 \mathrm{~h}$, so both applications can be used safely on human skin for in vivo evaluation.

Melanins are pigmented biopolymers that impart skin topology and tan synthesized by dendritic melanocytes dispersed at the dermo-epidermal junction. Tyrosinase is the key enzyme responsible for formation of melanins [10]. The bark is prosperous in phenolics viz. condensed tannin and phlobatannin, gallic acid, protocatechuic acid pyrocatechol, (+)-catechin, (-) epigallocatechin-7-gallate, and (-) epigallocatechin5,7 - digallate. The bark is also reported to contain (-) epicatechin, (+) dicatechin, quercetin, gallic acid, (+) leucocyanidin gallate, sucrose and $(+)$ catechin-5-gallate [7]. However, the inhibitory effect of catechins on melanogenesis has been reported, gallic acid inhibits melanin synthesis of B16 melanoma. Pyrogallol-containing compounds such as (-)-epigallocatechin-3gallate, (-)-epigallocatechin, gallic acid have significant inhibitory activity of melanin synthesis [1]. The decline in skin melanin can be credited to the catechins and gallic acid present in the active cream.

The inflammatory reaction following acute UV irradiation and the degenerative progressions associated with chronic UV radiation skin exposure are largely mediated by the overproduction of reactive oxidative species and by impairment of the antioxidant endogenous system $[6,11]$. UV-induced skin inflammation, oxidative stress and DNA damage etc. focus on mechanisms underlying the photo-protective effects of the phenolics [12]. A decline in the erythema level provided evidence against inflammation which leads to collagen deficiency and offer protection against photo-damage in our results of topically applied Acacia bark extract.
In the subjective analysis of applications after the panel test, the cream containing the Acacia extract proved to be acceptable and appropriate for topical use.

\section{Conclusions}

Our results show that the Acacia bark extract represents a valuable source of phenolic compounds produced naturally. Treatments of human skin with the active cream containing phenolic-rich Acacia bark extract significantly reduce skin melanin and erythema in vivo and could be used for topical treatment of hyperpigmentation and dermatitis. Furthermore, the good skin tolerance found after a single application in the patch test reinforces its accepted awareness as a topical antioxidant, after inclusion in appropriate and secure topical bases.

\section{References}

1. Sato K, Toriyama M. Depigmenting effect of catechins. Molecules 2009; 14: 4425-32.

2. Parvez S, Kang M, Chung HS, et al. Survey and mechanism of skin depigmenting and lightening agents. Phytother Res 2006; 20: 921-34.

3. Thorkil HE, Gislason T, Gislason D. Mite allergy and mite exposure in Iceland. Ann Agric Environ Med 2011; 18: 13-7.

4. Stankiewicz-Choroszucha BL, Wawrzyniak ZM, Lipiec A, et al. Consequences of smoke inhalation in the 'Epidemiology of Allergic Diseases in Poland' project (ECAP). Ann Agric Environ Med 2011; 18: 420-8.

5. Broniarczyk-Dyła G, Prusińska-Bratoś M, Kmieć ML. Assessment of the influence of licochalcone on selected functional skin parameters in patients with impaired vasomotor disorders and rosacea. Postep Derm Alergol 2011; 28: 241-7.

6. Almeida IF, Valentão P, Andrade PB, et al. In vivo skin irritation potential of a Castanea sativa (Chestnut) leaf extract, a putative natural antioxidant for topical application. Pharmacol Toxicol 2008; 103: 461-7.

7. Akhtar N, Waqas MK, Ahmed M, et al. Effect of cream formulation of fenugreek seed extract on some mechanical parameters of human skin. Trop J Pharm Res 2010; 9: 329-37.

8. Ali A, Akhtar N, Khan BA, et al. Acacia nilotica: a plant of multipurpose medicinal uses. J Med Plant Res 2012; 6: 1492-6.

9. Gaspar LR, Camargo FB Jr, Gianeti MD, Maia Campos PM. Evaluation of dermatological effects of cosmetic formulations containing saccharomyces cerevisiae extract and vitamins. Food Chem Toxicol 2008; 46: 3493-500.

10. Petit L, Pie GE. Skin-lightening products revisited. Inter J Cosmetics 2003; 12: 169-81.

11. Bissett DL. Common cosmeceuticals. Clin Dermatol 2009; 27: 435-45.

12. Nichols JA, Katiyar SK. Skin photoprotection by natural polyphenols: anti-inflammatory, antioxidant and DNA repair mechanisms. Arch Dermatol Res 2010; 302: 71-83. 